\title{
Recientes resultados de la investigación en el sitio arqueológico Puchuni, Atavillos Alto, Valle de Chancay
}

\author{
Recent Research Findings in the Archaeological \\ Site Puchuni, Atavillos Alto, Valley Chancay \\ Diana Antezana Ruiz, María Díaz Bejarano,* \\ Gabriela Contreras Ampuero, ${ }^{* * *}$ Augusto Neyra Carrillo ${ }^{* * *}$
}

http://dx.doi.org/10.21503/CienciayDesarrollo.2008.v9.03

\section{RESUMEN}

La investigación presente se llevó a cabo para reconocer el sitio arqueológico de Atavillos Alto, con el objetivo de determinar los tipos de arquitectura que se desarrollaron en el lugar denominado como Puchuni. Asimismo, tuvo como finalidad establecer la secuencia de ocupación de que fue objeto en su devenir histórico, para lo cual se aprovechó, además de los tipos arquitectónicos del lugar, los restos de cerámica superficial encontrados. Finalmente, se trató de comprobar la presencia inca en Puchuni a partir de los vestigios arquitectónicos presentes.

Palabras clave: Atavillos Alto, Puchuni.

\section{ABSTRACT}

The investigation shows up it carried out to recognize the archaeological place of High Atavillos, with the objective of determining the architecture types that were developed in the place denominated as Puchuni. Also, it had as purpose to establish the occupation sequence that it was object in their to become historical, for that which took advantage, besides the architectural types of the place, the opposing remains of superficial ceramic. Finally it was about checking the Inca presence in Puchuni starting from the vestiges architectural present.

Key words: Atavillos Alto, Puchuni.

* Arqueóloga de la Universidad Nacional Mayor de San Marcos.

** Arqueóloga de la Universidad Nacional Mayor de San Marcos.

${ }^{* * *}$ Arqueóloga de la Universidad Nacional Mayor de San Marcos.

${ }^{* * * *}$ Arqueólogo de la Universidad Nacional Mayor de San Marcos. 


\section{INTRODUCCIÓN}

En la bibliografía arqueológica del departamento de Lima, se cuenta con muy poca información sobre los Atavillos. Muy pocos son los investigadores que han estudiado este curacazgo y su área de desarrollo. Las principales fuentes de información las encontramos en los Documentos de Visitas a la provincia de Canta de los años 1549 y 1553 , analizadas por María Rostworowski en 1978.

En la primera mitad del siglo pasado, el monseñor Pedro Villar Córdova recorre parte de lo que fueron los territorios de los Atavillos, describiendo los restos arquitectónicos, así como algunos otros materiales culturales, pero también recoge algunas historias y mitos de la zona. Este esfuerzo fue secundado más tarde por Teodoro Cassana Robles y Fernando Villiger, entre otros. Sin embargo, son pocos los trabajos arqueológicos realizados en esta zona, por lo que quedan muchas interrogantes sobre los Atavillos, las cuales no pueden ser resueltas sin un mayor estudio. Muchos de los sitios arqueológicos de la región aún presentan un buen estado de conservación, pues el huaqueo es mínimo, dado que entre los pobladores de la zona existe un sentido de respeto hacia estos sitios, considerados por ellos como sus gentiles o sus abuelos, antepasados de su comunidad, y de quienes descienden.

El presente artículo es el resultado del reconocimiento delas evidencias arquitectónicas en el sitio de Puchuni (ver plano 1), localizado en el distrito de San Pedro de Pirca, en Atavillos Alto. Tal reconocimiento nos permitió diferenciar la distribución espacial del asentamiento, proponiendo cuatro tipos arquitectónicos, los cuales tendrán que ser comparados con otros asentamientos de Atavillos. Este es un primer paso para realizar a futuro un estudio de patrón arquitectónico y de asentamiento en la zona.

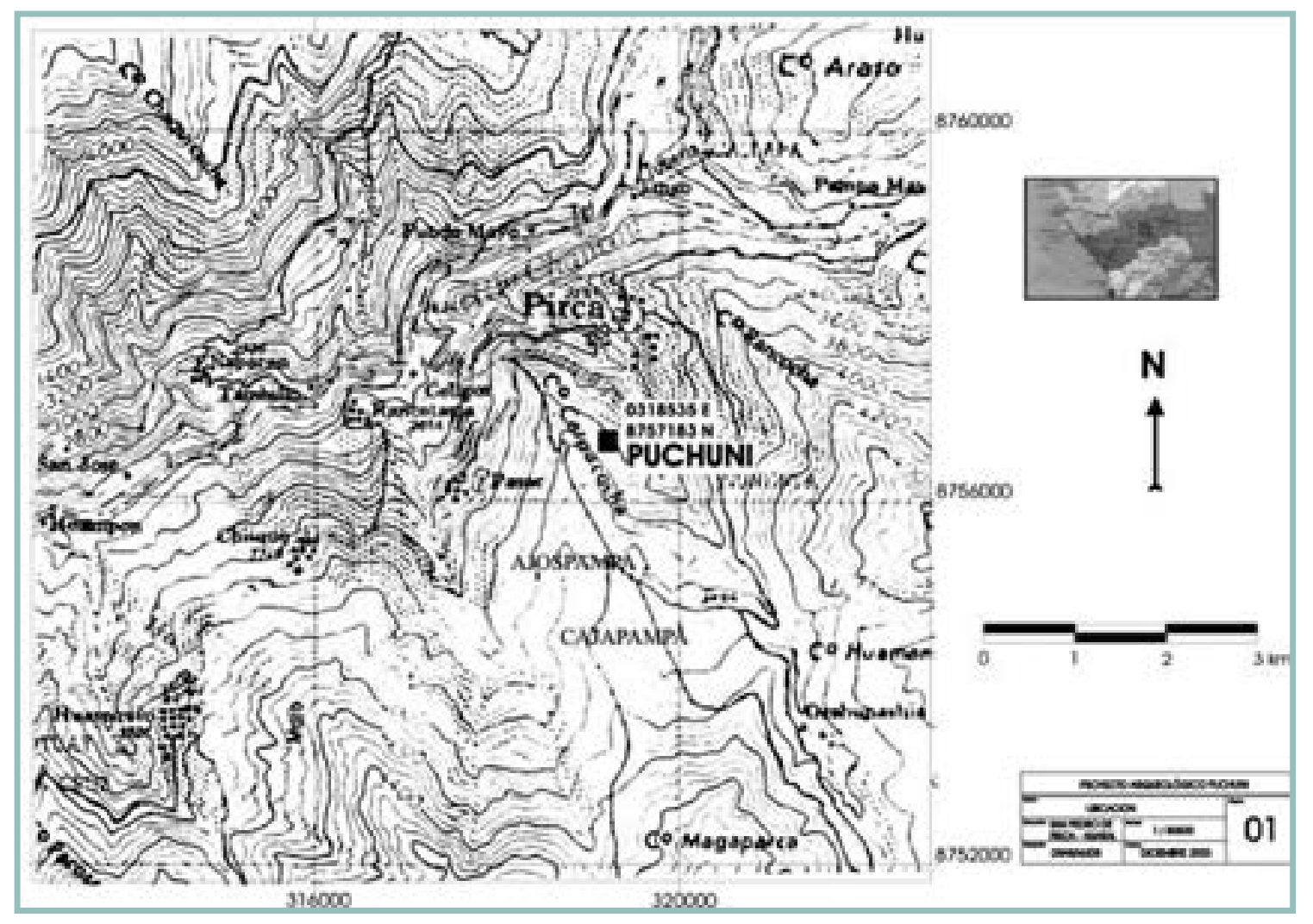

Plano 1 


\section{ANTECEDENTES}

En 1935, Pedro Villar Córdova realizó los primeros estudios de numerosos sitios arqueológicos que dejaron los primeros habitantes de Atavillos, en la antigua provincia de Canta (zona que actualmente corresponde a las provincias de Canta y Huaral). Según este autor, existieron en la sierra del departamento de Lima, antes del dominio incaico, dos agrupaciones sociales: los yauyos y los atavillos. Esa demarcación fue luego conservada por los incas, aplicándose el régimen de los "hunu", es decir, "provincia de 10000 tributarios". El hunu de los ata-willu comprendía la zona geográfica que actualmente ocupa la provincia de Canta, la sierra de Chancay y parte de Cajatambo. Esta antigua demarcación existió hasta la época de la conquista española, cuando se constituyeron los repartimientos, las encomiendas y después se formaron las provincias o corregimientos.

Posteriormente, María Rostworowski (1978) analiza los testimonios de las visitas de Canta de 1549 y de 1553 y da a conocer que el curacazgo de Atavillos se situaba en la margen izquierda del río Chancay hasta la margen derecha del río Chillón, dividido en bajo y alto; y que en tiempos prehispánicos consiguió el dominio de los cantas, piscas, huamantangas y socos. Según estos documentos, a la llegada de los españoles, el curacazgo de Canta seguía bajo la hegemonía de los Atavillos.

En cuanto al reconocimiento arqueológico de la región de los Atavillos, las investigaciones se han centrado en su arquitectura, pues muchos de sus asentamientos están en aceptable estado de conservación, tal es el caso de Añay, Chiprak y Rupac, ubicados en Atavillos Bajo. Estos sitios han sido estudiados y descritos por Fernando Villiger (1979), Teodoro Cassana Robles (1976) y F. Marussi Castellan (1979). Este último hizo un análisis urbanístico de la ciudad de Rúpac como tema de tesis, presentada al Centro de Estudios Urbanos en Madrid, en 1977.

Finalmente, Carlos Farfán Lobatón inició en el año 1984 recorridos por Cantamarca y Carcas, como parte de un proyecto auspiciado por el Instituto Nacional de Cultura. Además, realizó trabajos de excavación arqueológica en Cantamarca (temporadas 1987 y 1988) y en Huishco (temporadas 1989 y 1990). Durante las excavaciones arqueológicas recorrió ambas márgenes del río Chillón, y presentó los sitios arqueológicos reconocidos en varias publicaciones, haciendo énfasis en la descripción arquitectónica de los mismos, su ubicación cronológica así como las semejanzas y diferencias entre ellos.

Como podemos apreciar, existe poca información sobre los sitios arqueológicos de Atavillos, especialmente en la región de Atavillos Alto, donde la investigación arqueológica es casi nula. Al no realizarse mayores investigaciones arqueológicas, quedan muchas interrogantes sobre los grupos sociales que habitaron esta región, entre ellas, su ubicación temporal, el territorio que abarcaron, sus relaciones con otras etnias locales, si hubo o no presencia inca en los sitios de Atavillos, entre otras.

Es así que nuestro interés de investigación se centró en reconocer el sitio arqueológico Puchuni, ubicado en la región de Atavillos Alto, y para ello nos planteamos 1) especificar los tipos arquitectónicos que existen en el sitio de Puchuni, 2) establecer una secuencia de ocupación en base a los tipos arquitectónicos encontrados y a la recolección del material cerámico de superficie, y 3) definir la presencia inca en Puchuni a partir de los elementos arquitectónicos existentes en el sitio.

\section{UBICACIÓN}

El sitio arqueológico de Puchuni se ubica en la jurisdicción de San Pedro de Pirca, capital del distrito de Atavillos Alto, provincia de Huaral, 
departamento de Lima, en la margen izquierda del río Chancay. Su ubicación en coordenadas UTM es: E 318533 y N 8757183, a una altitud de 3350 m.s.n.m.

Puchuni está ubicado en los límites de los poblados de San Pedro de Pirca y Pasac. Su acceso se realiza a través del pueblo de Pasac, tras una hora de caminata ascendente por un sendero rodeado de exuberante vegetación arbustiva y espinosa, propia del piso ecológico (región Quechua: 2300 a 3500 m.s.n.m.).

\section{ENTORNO Y DESCRIPCIÓN DEL SITIO}

Puchuni ocupa un área por lo general plana del cerro Carpacocha, la misma que posee unas dimensiones $110 \mathrm{~m}$ de noreste a suroeste y de 350 $\mathrm{m}$ de noroeste a sureste $\left(38500 \mathrm{~m}^{2}\right)$. Al oeste del sitio arqueológico, sobre el mismo cerro, cruza el canal de Ocana, el cual divide los poblados de San Pedro de Pirca y Pasac.

Es un asentamiento de forma alargada (ver figura 1), con una orientación noroeste-sureste y una pendiente de suroeste a noreste, siguiendo la ladera del cerro Carpacocha. En la actualidad, la zona es netamente ganadera (ganado vacuno) pero sin dejar de trabajar el campo, para lo cual reutilizan los amplios terrenos aterrazados y los andenes que existen en la zona, construidos desde épocas prehispánicas. La vegetación cultivada está integrada básicamente por maíz, habas, alfalfa y papas sin riego, aunque esta actividad agrícola se da en forma temporal.

En el sitio se observan grandes edificaciones de piedra cubiertas por vegetación espinosa y arbustiva (Phylum Lepido y otras del género Lupinus), lo cual hizo necesario una primera intervención en el sitio para realizar trabajos de desyerbado en algunas de las estructuras. Esto nos permitió ejecutar el levantamiento arquitectónico del sitio, pudiendo identificar cuatro sectores bien definidos.

SECTOR I: Se ubica en el lado noroeste del sitio y consiste en una serie de espacios abiertos, generalmente de forma alargada e irregular, con fuerte pendiente de suroeste a noreste, siguiendo el contorno del cerro Carpacocha. Estos espacios son aterrazados, ya que poseen muros de contención en sus límites, y en otros casos se han levantado muros con la técnica constructiva del pircado, que van de 1,00 a $1,60 \mathrm{~m}$ de altura y $0,70 \mathrm{~m}$ de espesor en promedio, lo cual no permite el acceso directo. En la actualidad, estas áreas son utilizadas como campos de cultivo por los pobladores de San Pedro de Pirca.

SECTOR II: Es el área monumental del sitio (ver figura 2) donde se ubican grandes edificios de piedra canteada de aproximadamente 4,00 $\mathrm{m}$ de altura en promedio, con vanos de acceso pequeño que nos permiten ingresar a cuartos interiores. Sobresale en este sector un edificio que posee dos grandes vanos de forma trapezoidal que van desde el suelo hasta la cubierta (ver foto $\left.\mathrm{N}^{\circ} 10\right)$. Este tipo de estructura fue reportado por Villar Córdova (1935) en el sitio de Chiprak y Rúpac en Atavillos Bajo.

Los edificios en general han sido edificados sobre tres terrazas artificiales que van a lo largo de todo el sector. Estas terrazas constructivas son

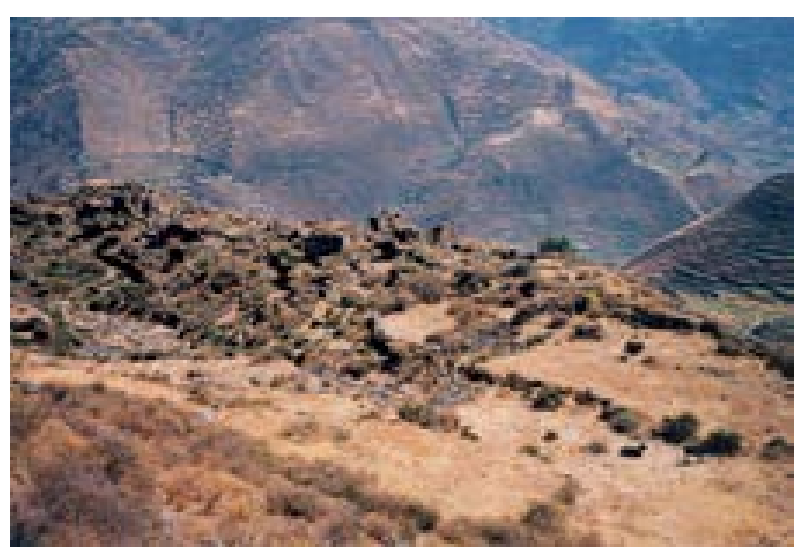

Figura 1 


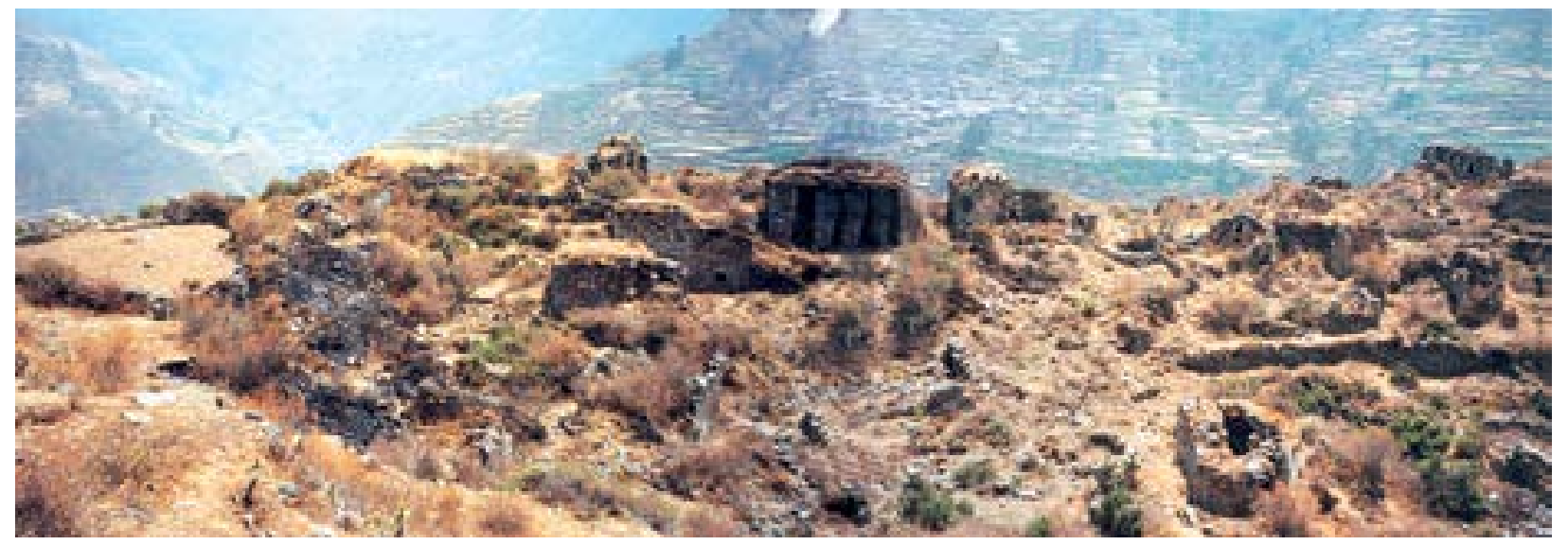

Figura 2

llenadas con gran cantidad de piedras y cubiertas por tierra, para nivelar el terreno. Entre los edificios se dejaron espacios abiertos.

SECTOR III: Consiste en una serie de terrazas (ver figura 3) ubicadas en la ladera del cerro Carpacocha, hacia el lado oeste del sitio y al este del canal de Ocana. Las terrazas tienen forma alargada y siguen la orientación del sitio con una fuerte pendiente hacia el noreste. Se utilizan muros de contención de 2,00 a 3,00 $\mathrm{m}$ de alto aproximadamente, los cuales sirven para nivelar el terreno. En este sector, llama la atención una plaza alargada de gran dimensión, delimitada por muros con aparejo bien logrado. En la esquina sureste de dicha plaza se distingue una estructura derrumbada de poca altura, en la cual se ubica un canal de 2,47 m de largo y 0,16 $\mathrm{m}$ de ancho. Este espacio abierto se encuentra en una parte alta, a $20 \mathrm{~m}$ de distancia del canal de Ocana.

SECTOR IV: Se ubica en el lado sureste del sitio. Es un área de espacios abiertos delimitados con muros de poca altura, similares al Sector I. En la actualidad, estos espacios son utilizados como pastizales para el ganado.

\section{TIPOS ARQUITECTÓNICOS}

Para el análisis arquitectónico se elaboró un plano detallado del Sector II (ver plano 2), y conjuntamente con el registro de fichas de

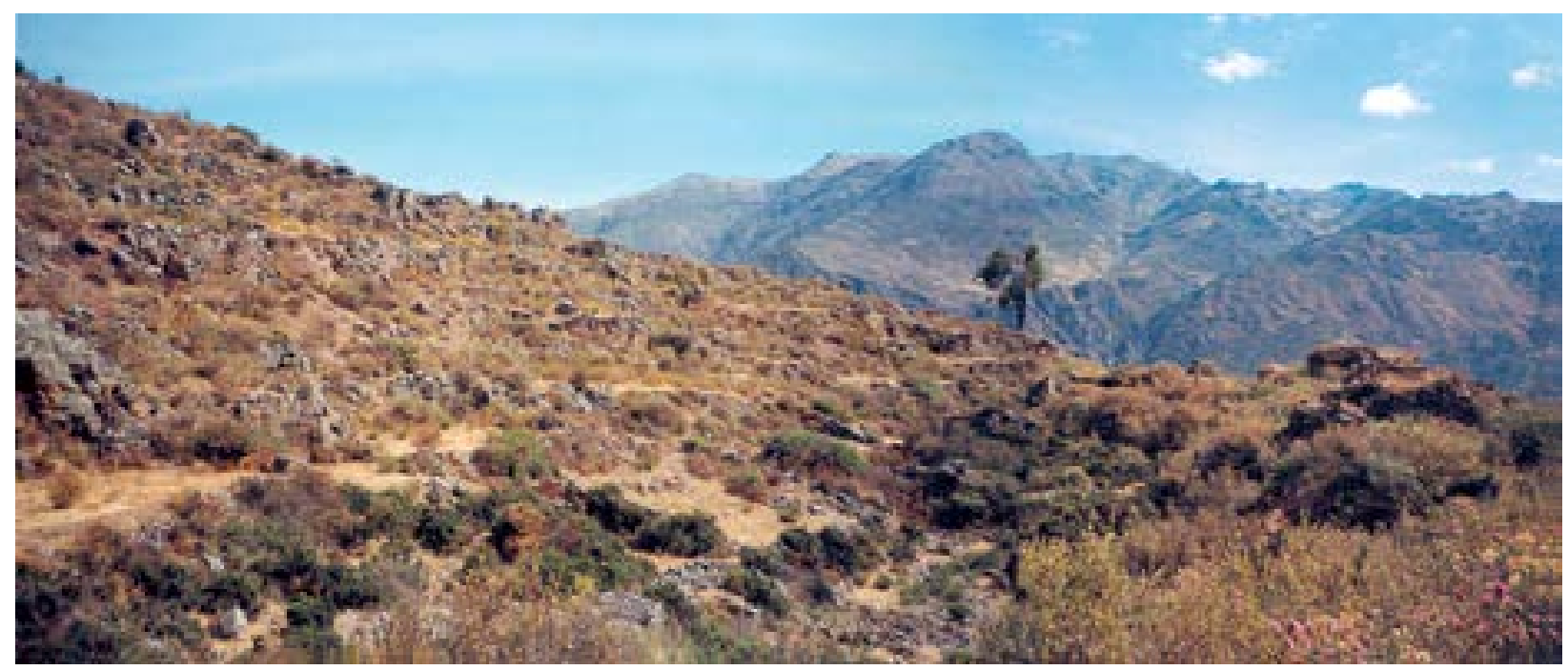

Figura 3 


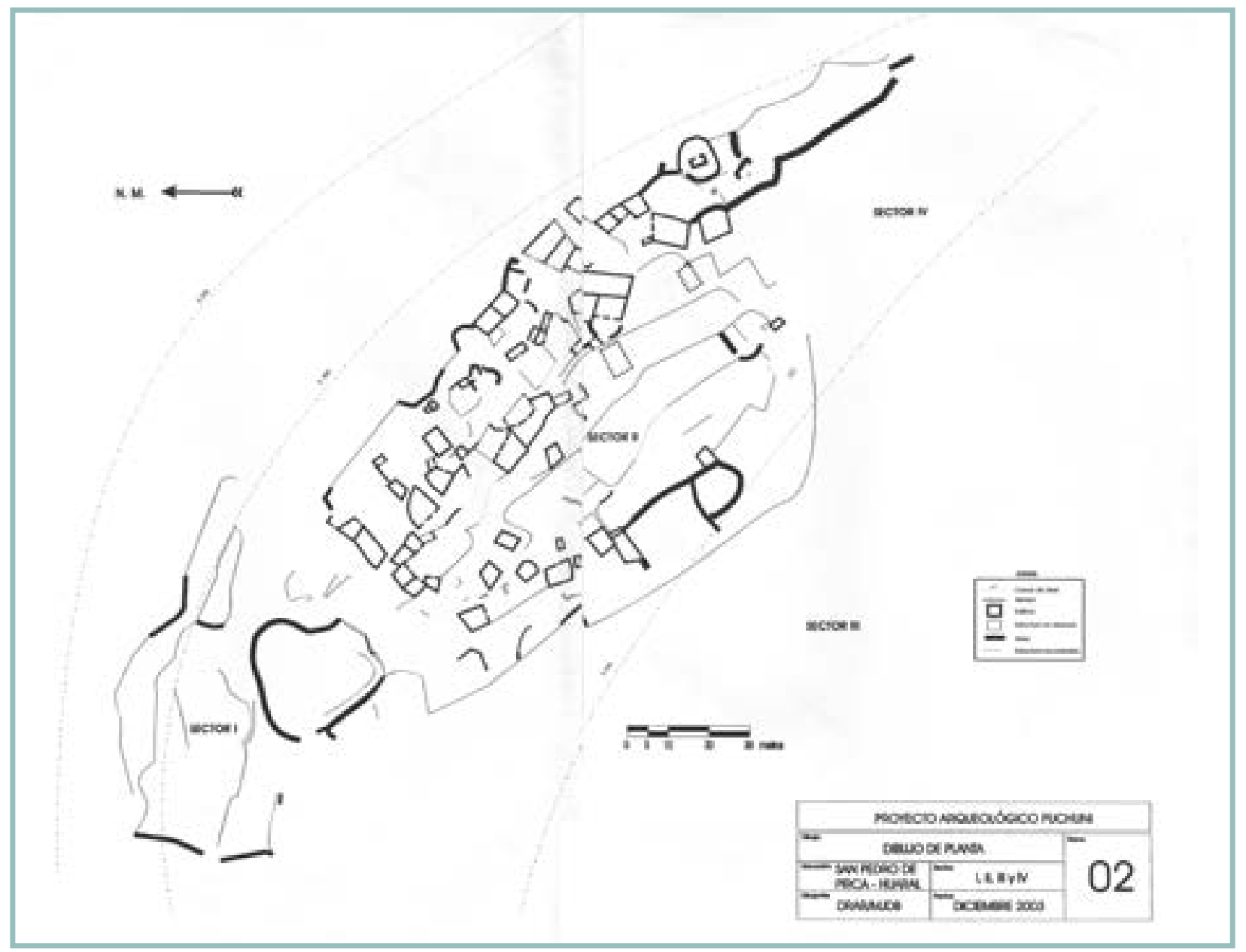

Plano 2.

arquitectura se identificaron edificios, espacios abiertos y terrazas constructivas. Solamente se registró el $33 \%$ del total de cien estructuras, priorizándose aquellas que se encontraban en buen estado de conservación. Antes de describir nuestra tipología, definiremos los términos utilizados ${ }^{3}$ para nuestro análisis.

Alacena: Cavidad en el espesor de los muros. Presenta vanos trapezoidales y rectangulares de $0,40 \mathrm{~m}$ de ancho por $0,60 \mathrm{~m}$ de alto, y un espacio interno de $0,80 \mathrm{~m}$ de ancho, $1,50 \mathrm{~m}$ de largo y $1,00 \mathrm{~m}$ de alto en promedio. Se distribuye en el tercio medio y superior de los muros internos del edificio (ver figura 4).

Cartela: Miembro voladizo sobre el que se asienta la ménsula, de más altura que vuelo (ver figura 5).
6). $\quad$ Cubierta: Techo de un edificio (ver figura

Edificio: Llamamos edificio a una estructura techada, con muros de $4,00 \mathrm{~m}$ de alto en promedio, en cuyo interior se encuentran dos o más cuartos pequeños. En el frontis de estos edificios se observa una ventana pequeña sobre el vano de acceso. El primer cuarto al que se accede por el vano principal tiene una planta cuadrangular u ovalada, y presenta los siguientes elementos: alacenas, nichos, pilastras, cartelas, ménsulas. En algunos casos se hallaron restos de enlucido rojo.

Espacios abiertos: Son estructuras sin cubierta, limitadas por muros. Estos espacios pueden ser patios, plazas o terrazas. Estas últimas son de carácter constructivo, lo cual permite la 


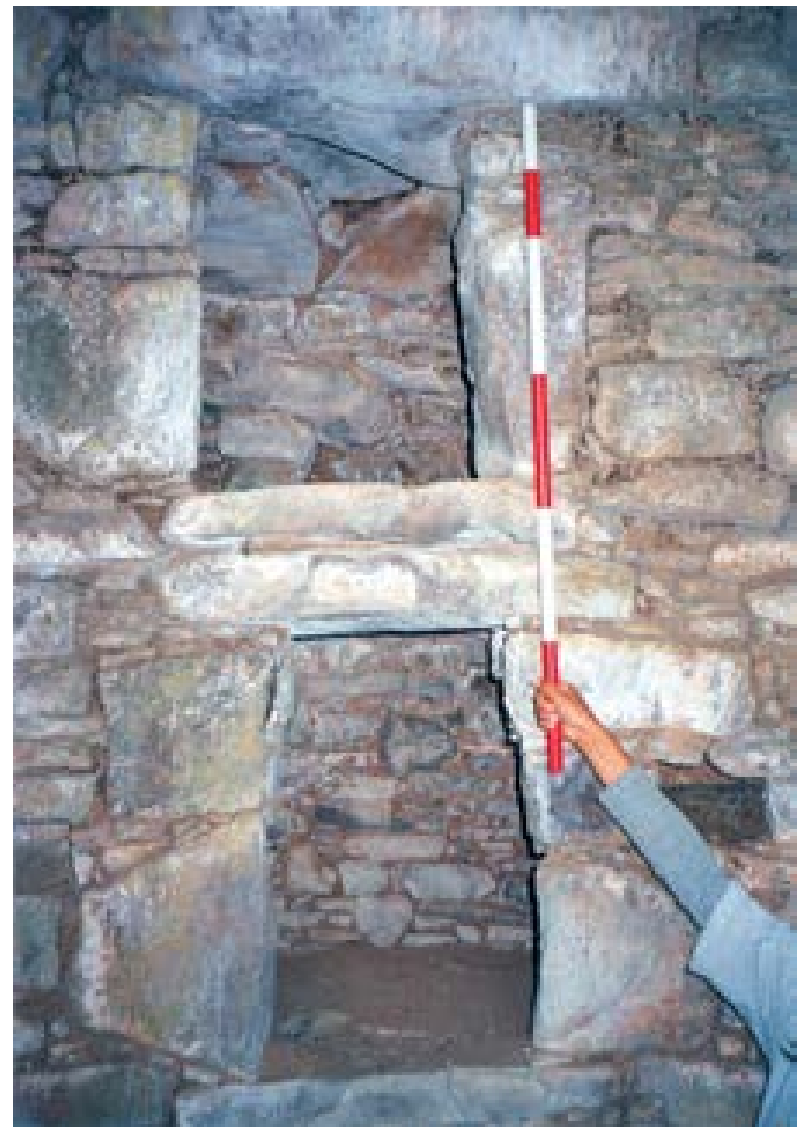

Figura 4.

nivelación de terreno, con el único propósito de construir edificios que solos o en su conjunto forman espacios abiertos.

Ménsula: Miembro de arquitectura que sobresale de un plano vertical y sirve para recibir o sostener alguna cosa (ver figura 5).

Nicho: Cavidad en el espesor de un muro, de menor tamaño que una alacena (ver figura 7).

Mampostería: Piedras canteadas dispuestas en hiladas irregulares y cuñas de piedras menores unidas con argamasa de barro.

Pilastra: Columna de forma trapezoidal invertida, adosada en la parte baja de los paramentos internos. Funcionan como soporte del adelantamiento del muro, además de dividir espacios y ser un elemento decorativo (ver figura 8).

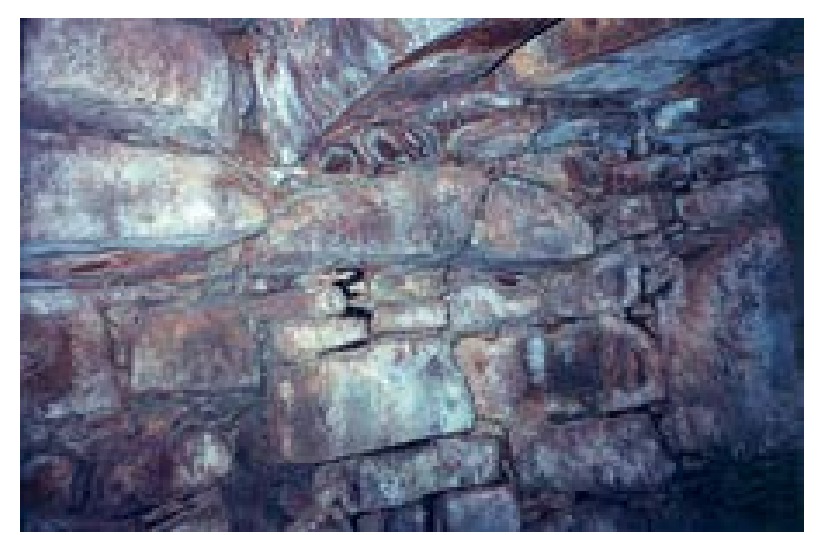

Figura 5

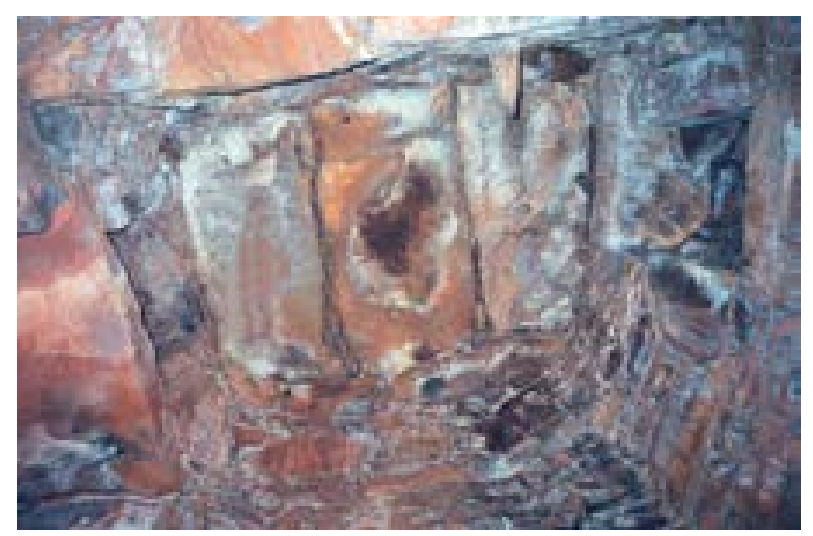

Figura 6

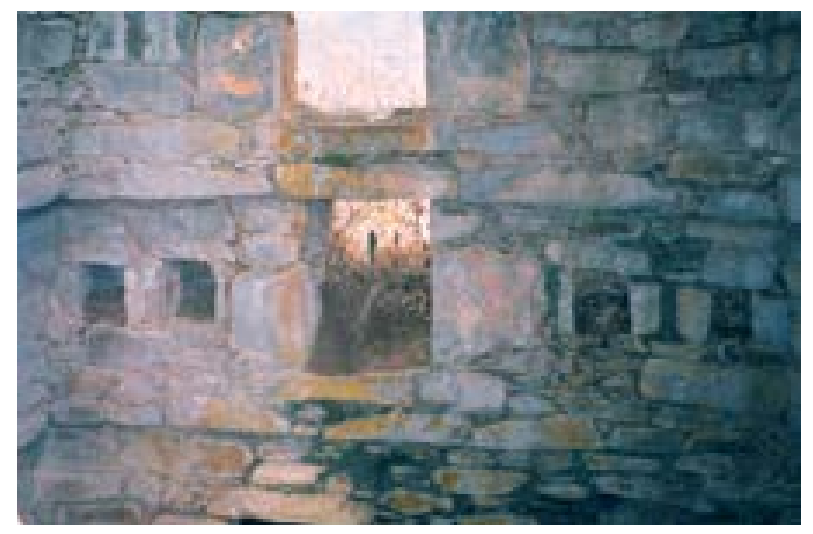

Figura 7

Pircado: Piedras sin cantear, colocadas y ajustadas unas con otras, sin formar hiladas y sin utilizar argamasa.

Terrazas: Espacios abiertos de terreno llano, dispuestos en forma de escalones en la ladera del cerro.

Vestíbulo: Atrio que está a la entrada de un edificio. 


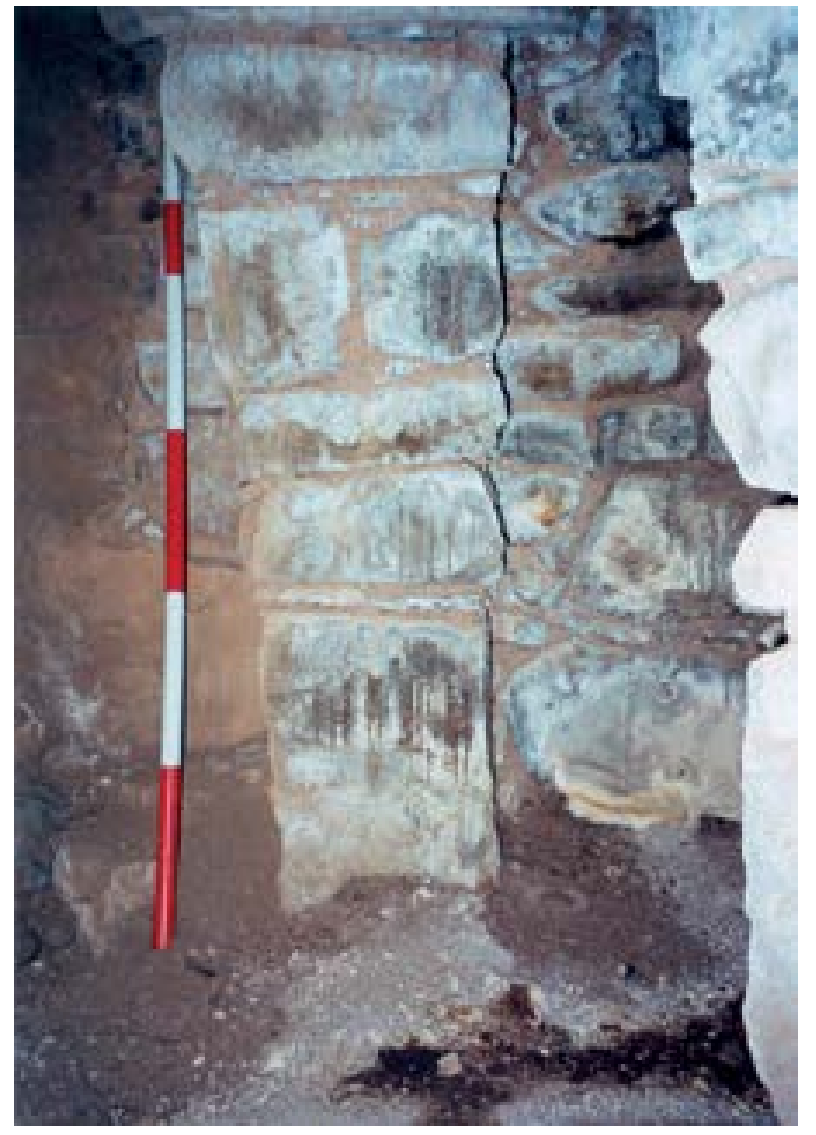

Figura 8

La técnica constructiva utilizada en el sitio para los edificios consiste en piedras canteadas dispuestas en hiladas irregulares y cuñas de piedras menores unidas con argamasa de barro. Los edificios y espacios abiertos se relacionan formando tipos arquitectónicos, de los que hallamos cuatro en Puchuni. Así tenemos:

TIPO 1. Es un edificio aislado de $3,50 \mathrm{~m}$ de altura y planta rectangular, con un vano de acceso de $0,50 \mathrm{~m}$ de ancho y $0,70 \mathrm{~m}$ de alto, $\mathrm{y}$ una ventana pequeña en el frontis de $0,15 \mathrm{~m}$ de ancho y $0,20 \mathrm{~m}$ de alto (ver figura 9). Su interior está constituido por un cuarto principal que tiene una altura casi igual a la del edificio y uno o más cuartos pequeños de menor altura ubicados en la parte posterior. Desde la parte inferior, los muros van adelantándose por nivel, de manera que se va reduciendo el espacio interno del cuarto hasta llegar a la cubierta. Distribuidos en todos los muros, se encuentran alacenas, nichos, y en algunas ocasiones pilastras y cartelas.
La cubierta por el interior está formada por lajas grandes dispuestas en un solo sentido y cubren un espacio ovalado interno. En la parte superior de los muros se ubican ménsulas sobre las cuales se depositan estas lajas.

TIPO 2. Se trata de un edificio de $4,00 \mathrm{~m}$ de altura, con dos vanos de acceso de forma trapezoidal que van desde el nivel del suelo hasta llegar a la cubierta (ver figura 10). Presenta un vestíbulo que da acceso a dos cuartos independientes con las mismas características internas del tipo 1. A este se le asocia un espacio abierto exclusivo que puede estar limitado por muros de 0,80 m de alto. Por lo general, este tipo de edificio se va a encontrar en un nivel más elevado que el tipo 3 y 4 .

TIPO 3. Está representado por un edificio de 4,00 $\mathrm{m}$ de altura y presenta dos o más cuartos independientes, formando una sola estructura (ver figura 11). Los cuartos independientes son

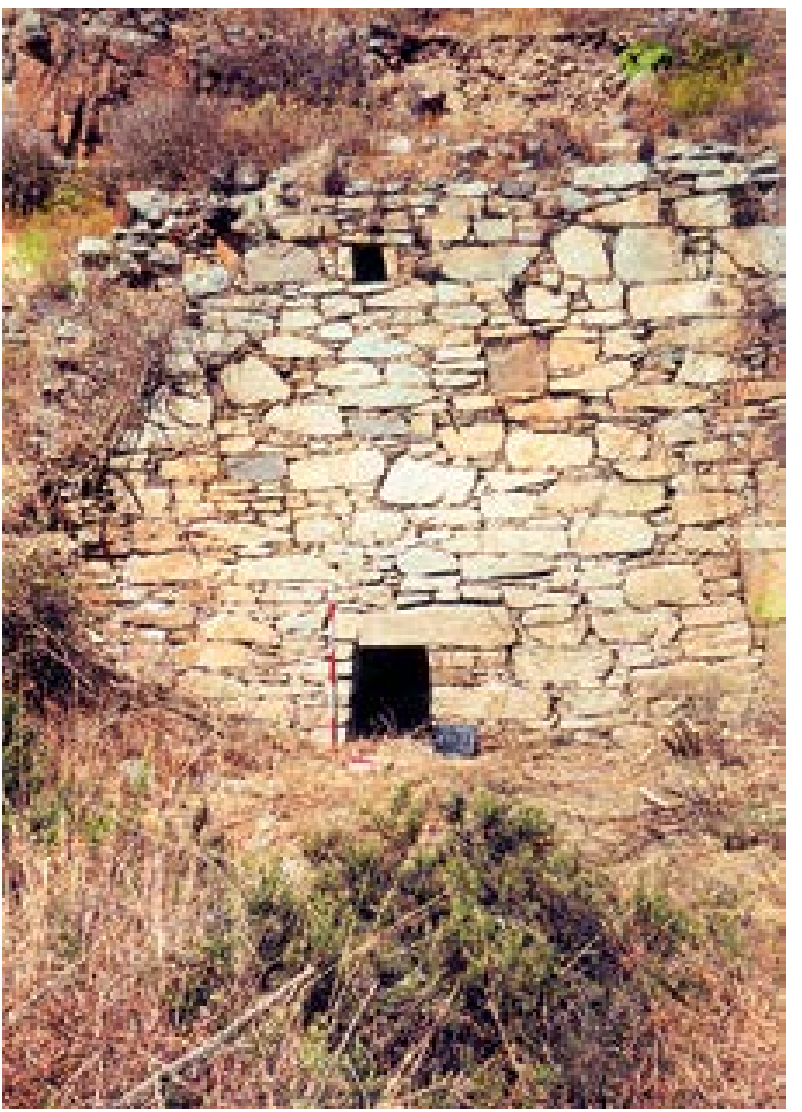

Figura 9 


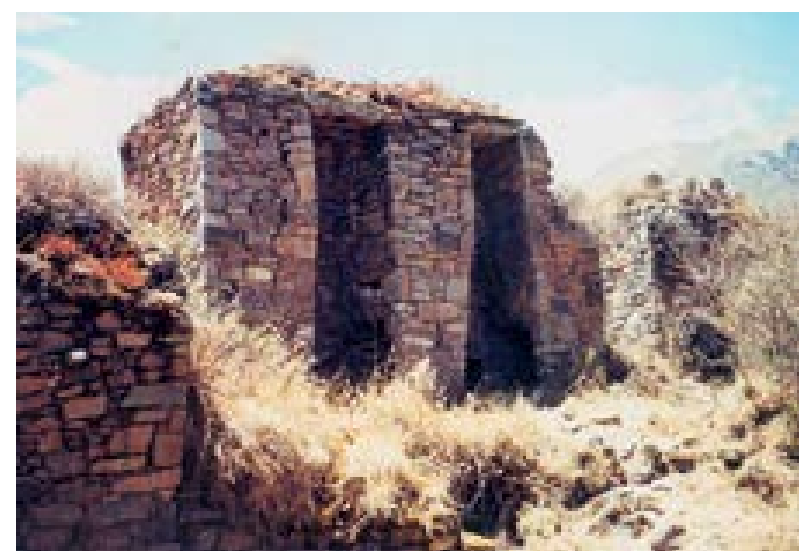

Figura 10

similares en su interior al tipo 1. Los vanos de acceso dan a un patio común limitado por los mismos edificios. Este tipo de estructura se ubica en las terrazas constructivas II y III, en un nivel por debajo de la superficie de estas terrazas. Asociada a este tipo se encuentran edificios pequeños de $1,30 \mathrm{~m}$ de altura.

TIPO 4. Es un conjunto de edificios asociado a un espacio abierto común que se forma por los límites de los mismos y también presenta edificios pequeños de $1,30 \mathrm{~m}$ de altura en promedio (ver figura 12). Es en este tipo arquitectónico que se halló un aríbalo pequeño, en una de las estructuras que lo conforman.

\section{CONCLUSIONES}

Puchuni se ubica en una zona plana del lado este del cerro Carpacocha, que ha sido modificada a través de tres terrazas artificiales sobre las cuales se construyeron los edificios, ocupando tierras aptas para el cultivo. No presenta murallas, a diferencia de otros sitios reportados como Rúpac, Chiprak y Añay (Atavillos Bajo), pudiéndose ingresar al sitio desde cualquier punto. Además, no tiene un dominio visual del valle, como es el caso de los sitios antes mencionados.

El resultadode nuestro análisis arquitectónico nos lleva a identificar cuatro sectores bien diferenciados. Los sectores I y IV, compuestos de

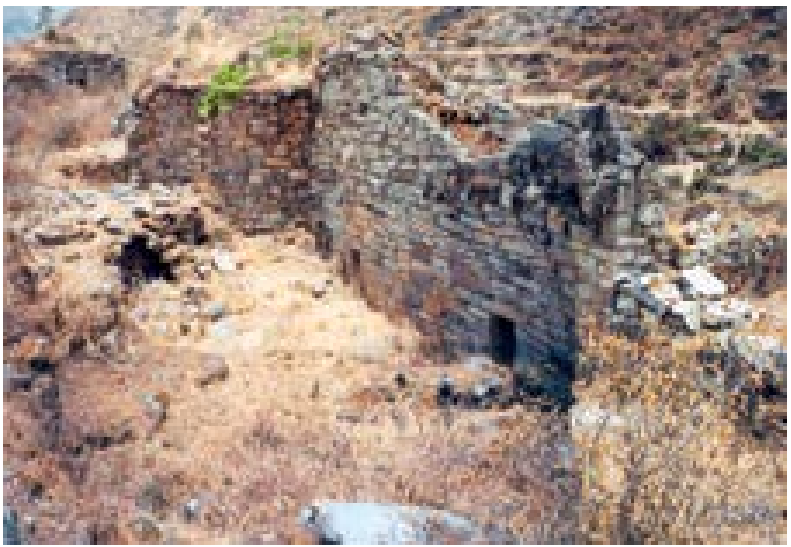

Figura 11

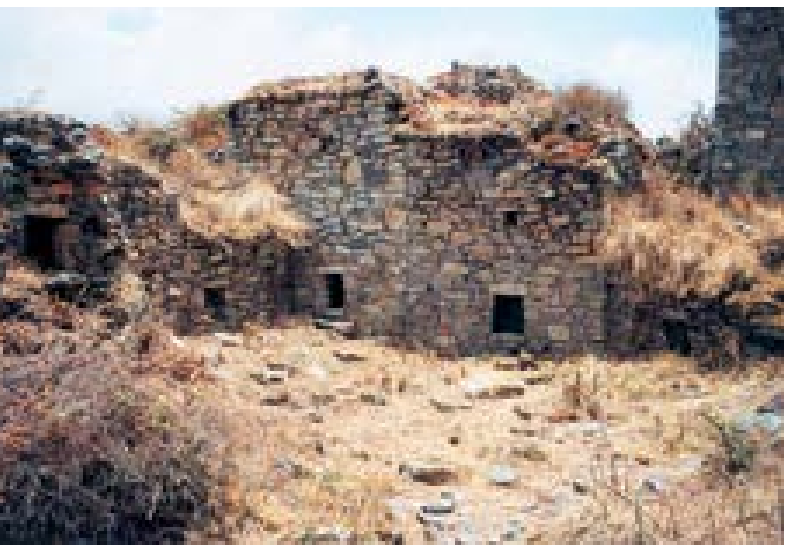

Figura 12

espacios abiertos aterrazados y algunos cercados con muros bajos, han podido ser utilizados como corrales temporales o áreas de cultivo que rodeaban el sector II, correspondiente al área residencial de Puchuni. El sector III presenta igualmente terrazas; sin embargo, estas tienen una mayor pendiente y forma alargada, siguiendo las curvas del nivel del cerro Carpacocha, además de presentar restos de arquitectura aislada.

Por presentar una mayor cantidad de estructuras y ser el área residencial, nos centramos en la descripción y análisis del Sector II, en donde identificamos cuatro tipos arquitectónicos en los que se realizaban diferentes actividades. En el tipo 1 es posible que se realizaran actividades de un grupo reducido, posiblemente de índole familiar y en un solo espacio habitacional aislado. No se puede especificar la actividad ahí realizada por falta de evidencias en superficie. 
El tipo 2 puede corresponder a la misma actividad realizada en el tipo I, pero por un estrato social más alto, ya que comprende un edificio en un área restringida de mayor tamaño y que cuenta con variedad de elementos arquitectónicos que le dan un mejor acabado, lo que indica una mayor dedicación en la construcción de estos edificios.

Los tipos 3 y 4 corresponden a áreas comunales donde se habrían realizado diferentes actividades domésticas y grupales que mantienen independencia en cuanto a ocupar un edificio habitacional, pero compartiendo un espacio abierto común.

Al parecer, todas las estructuras interiormente tuvieron enlucido rojo, y a su vez se hallaron en ellas restos óseos humanos, lo cual nos indica una actividad funeraria, pero que no es exclusiva de algún tipo arquitectónico.

A partir del análisis arquitectónico del sitio, no se encontró arquitectura de los estilos inca, kallanca, kancha, colca y otros que nos indiquen su presencia en Puchuni. Sin embargo, los fragmentos de cerámica recolectados en superficie son en su mayoría formas del Horizonte Tardío, de manufactura local, a excepción de un aríbalo pequeño.

Esta ausencia de arquitectura de estilo inca también es reportada por Mercedes Cárdenas (1977) para el valle de Huaura. Ella establece que durante el Horizonte Tardío disminuye la evidencia de ocupación inca, lo que estaría indicando que los grupos del Intermedio Tardío siguieron coexistiendo y manteniendo su propia cultura hasta la llegada de los españoles, al lado de los rasgos culturales traídos por los incas. Krzanowski (1991) manifiesta también que el hecho de que en los valles de Chancay y Huaura hasta la fecha no se haya encontrado ningún sitio inca no significa que no existiera. Sin embargo, es un argumento para afirmar que la ocupación por los incas tenía un carácter pacífico y que su gobierno se apoyaba en la élite local. Esta situación se habría presentado en Puchuni, ya que se evidencia una presencia inca manifestada en la cerámica mas no en la arquitectura del sitio, la cual presenta técnicas constructivas locales similares a Rúpac, Chiprak y Añay.

Esta presencia inca y ausencia de su arquitectura nos estaría indicando una forma de gobierno indirecto, del tipo de gobierno identificado para el valle del Chillón, específicamente entre los chacllas e incas (Silva, 1988). El gobierno indirecto se caracteriza por establecer alianza entre el Inca y el gobierno local, evitando la presencia directa del Inca. Esta alianza reportaba dádivas al gobierno local y tributo al gobierno inca.

La cronología relativa del sitio de Puchuni, basada en la cerámica con formas incas y un fragmento de botella con decoraciones incisas identificadas como estilo Quillahuaca, de difusión en el Intermedio Tardío (Krzanowski, 1986), nos da un fechado entre el Intermedio Tardío (1200 a 1440 d. C.) y el Horizonte Tardío (1440 a 1532 d. C.). Por lo tanto, el sitio arqueológico Puchuni es un sitio preconcebido y planificado para períodos tardíos, con técnicas constructivas de los atavillos, por lo que inferimos que fue construido por esta sociedad.

Puchuni, al estar ubicado en los límites de los pisos ecológicos Quechua y Suni (2300 a 3500 / 3500 a 4000 m. s.n.m.), domina una gran variedad de ecosistemas y una vasta gama de producción. A su vez, su ubicación en medio de terrazas de cultivo nos estaría indicando que fue un centro de control de estas áreas durante este periodo.

Latipologíaquehemos presentadoes exclusiva del sitio arqueológico Puchuni; sin embargo, esperamos encontrar esta misma tipología en los diferentes sitios arqueológicos ubicados en esta región. Este es entonces un trabajo preliminar para lo que podría ser un Patrón Constructivo para los sitios de los Atavillos. 


\section{REFERENCIAS BIBLIOGRÁFICAS}

1. Cárdenas Martín, Mercedes.

1977. Informepreliminar del trabajo de campo del valle de Huaura (departamento de Lima). PUCP, Instituto Riva Agüero, Seminario de Arqueología, Lima.

2. Casana Robles, Teodoro.

1976. Restos arqueológicos de la provincia de Canta y la provincia de Huaral. Lima, Imprenta Colegio Militar Leoncio Prado. 238 pp.

3. Farfán Lovatón, Carlos.

1988. "Informe preliminar de los trabajos arqueológicos en Cantamarca". Actas y Trabajos del VI Congreso Peruano: Hombre y Cultura Andina. F. Iriarte B., editor, tomo 3, pp. 147-167. Lima, Facultad de Ciencias Sociales de la Universidad Inca Garcilaso de La Vega y Consejo Nacional de Ciencia y Tecnología.

1992. "Los asentamientos prehispánicos de altura y su relación con el espacio geográfico en la cuenca alta del Chillón”. En: Boletín de la Sociedad Geográfica de Lima. Segunda Época, vol. 105.

1995. "Asentamientos prehispánicos en la cuenca alta del río Chillón". En: Gaceta Arqueológica Andina, No 24, pp. 31-61. INDEA.

4. Gasparini, Graciano y Luise Margolies.

1977. Arquitectura Inka. Universidad Central de Venezuela, Facultad de Arquitectura y Urbanismo.

5. Krzanowski, Andrzej.

1986. Cayash prehispánico. Primera parte del informe sobre las investigaciones arqueológicas de la expedición cientifica polaca a los Andes. Proyecto Huaura - Checras (Perú, 1978).

1991. "Influencia inca en los valles de Huaura y Chancay”. En: Estudios sobre la cultura Chancay. Perú, Krakow, Polonia.

6. Marussi Castellan, F.

1979. "Rúpac: análisis urbanístico de una ciudad prehispánica”. Boletín del Instituto Francés de Estudios Andinos 8 (1-2). Lima. pp. 1-33.

7. Marcus, Joyce y Jorge Silva Sifuentes.

1988. "Los cocales del valle de Chillón. Evidencia arqueológica y contexto ecológico". En: Conflicts Over Coca Fields in XVIthCentury Perú, by María Rostworowski, con la colaboración del Instituto de Estudios Peruanos. Vol. IV.

8. Rostworowski, María.

1978. Señorios indígenas de Lima y Canta. Lima, Instituto de Estudios Peruanos. 280 pp.

9. Villar Córdova, Pedro.

1933. "El mito de Wa-Kon y los willka referente al culto indígena de la Cordillera de La Viuda”. Revista del Museo Nacional 2(2). Lima.

1940. Las culturas prehispánicas del departamento de Lima. Lima.

1940. "Sobre las chullpas de la provincia de Canta". En: Revista Chasqui, Vol.I, No2. Órgano de la Asociación Peruana de Arqueología.

10. Villiger, F.

1979. "Hay una fortaleza precolombina escondida". Boletín de Lima No 1. Lima. pp. 53-57. 